\title{
Realization of Initiative Repair of Power Distribution Network Based on Backpropagation Neural Network Optimization
}

\author{
Zhihua Guo, ${ }^{1} \mathrm{Na} \mathrm{Li},{ }^{1}$ Dongping Qiao, ${ }^{*}{ }^{*} \mathrm{Hu}$ Qiao, ${ }^{1}$ and $\mathrm{Chao} \mathrm{He}^{2 * *}$ \\ ${ }^{1}$ State Grid Qinghai Electric Power Co., Ltd., Qinghai 810000, China \\ ${ }^{2}$ School of Communication and Information Engineering, \\ Chongqing University of Posts and Telecommunications, Chongqing 400065, China
}

(Received July 1, 2021; accepted October 11, 2021)

Keywords: power distribution network, fault diagnosis and prediction, backpropagation neural network, initiative repair, intelligent sensing devices

Protection and maintenance systems are necessary for transmission systems to ensure an efficient and reliable power supply when faults occur. However, most fault detection and location methods rely on the electricity measurement provided by current and voltage transformers. In addition, the initiative repair of a power distribution network is a very considerable portion of power grid operation, and the initiative repair efficiency is crucial for power supply enterprises to provide high-quality services. To give full attention to the value of perceived data and improve the accuracy of fault diagnosis, the technical performance of high-speed power line carriers is extensively investigated, and intelligent sensing devices are installed in power distribution network lines, stations, branch boxes, table boxes, and other parts to realize the initiative repair. To improve the accuracy and timeliness of fault diagnosis for the power distribution network, in this paper, we propose an initiative repair and judgment mechanism based on backpropagation (BP) neural network optimization for the power distribution network. After acquiring information data such as power consumption, the mechanism first takes advantage of the filtered data information to train the BP neural network to predict fault information and other data. Finally, the initiative repair of the power distribution network obtains the forecast data of the BP neural network to judge the fault of the power distribution network. The proposed mechanism considerably shortens the time for the initiative repair and improves the traditional repair mode of the power distribution network, thereby improving the initiative repair efficiency of the power distribution network.

\section{Introduction}

Owing to the complexity of the power distribution network, both power distribution and protection are rigorous challenges for engineers. The basic function of protection is to ensure the supply continuity of the whole system and minimize equipment damage by detecting faults. ${ }^{(1)} \mathrm{A}$ good protection system must ensure the reliability of the system by protecting the operation of

\footnotetext{
*Corresponding author: e-mail: syyxzzbjb@126.com

${ }^{* *}$ Corresponding author: e-mail: d170101004@stu.cqupt.edu.cn https://doi.org/10.18494/SAM.2021.3515
} 
the system against all types of failure at all locations in the network. The system must be secure and reliable. In the power distribution network, overload, overvoltage, power fluctuations, and other reasons will cause a fault. When a fault occurs, the protective device starts the circuit breaker operation, disconnecting the power to the faulty part. In general, a circuit breaker is one of the main pieces of equipment in the network fault protection scheme. This protection must be studied and judged before overcurrent and overvoltage, which can cause damage to the connected equipment, such as transformers. Therefore, rapid fault detection can help protect equipment, allowing damage to be reduced before disconnecting a faulty line. On the other hand, accurate fault location can help power company workers troubleshoot persistent failures and locate areas where failures often occur, thereby reducing the frequency and duration of outages. Thus, while fault detection and location schemes have been developed, various algorithms are still being developed to perform this task more accurately and efficiently.

Owing to its different topology and operation characteristics, the fault location of the power distribution network still faces many challenges. The traditional fault location method of the power distribution network includes the visual inspection of power line patrol. For underground cabling systems, faults are also located by a dedicated cable test vehicle. However, these traditional fault location methods cannot quickly locate the fault. The increasing dependence of the power distribution network on electricity requires a reliable supply of electricity. Recently, regulators have required utilities to meet system performance benchmarks. ${ }^{(2)}$ Therefore, this paradigm has driven the demand for intelligent distribution systems, which are very critical and useful for the detection, location, and classification of a power distribution network.

In recent years, various fault detection schemes have been developed for the transmission network, but the accurate location of faults has not been considered, and the faults in the power transmission network have not been classified. Therefore, to improve the service quality of power supply, enhance the efficiency and accuracy of fault analysis, and further transform distribution services from passive to active, the initiative repair of the power distribution network based on the backpropagation (BP) neural network is proposed. The power distribution network achieves an initiative repair mechanism, a fusion energy management, control, and electricity information acquisition system, distribution automation, an equipment operations lean management 2.0 system, a geographical information system, fault indicator data of the master station system, and BP neural network prediction. The realized information is used for the transformation of the traditional repair mode, which contributes to realizing active and efficient fault investigation and initiative repair.

\section{Current Research on Initiative Repair of Power Distribution Network}

Recently, the initiative repair project has been transformed into process management, the standardization in management level, and a militarized form in terms of on-site initiative repair. ${ }^{(3)}$ To streamline the process of users' fault reporting to initiative repair, the command platform of the power distribution network under the initiative repair project is fully utilized. Through the communication between the mobile transceiver equipped by the initiative repair team and the initiative repair command platform, the initiative repair of the power distribution 
network is supplemented. ${ }^{(4)}$ From the analysis of the current initiative repair process, the limitations of the power distribution network are as follows:

(1) After the occurrence of a power failure, it will take a long time from the user's perception of the fault to the call for initiative repair, which highlights the weakness that the power supply enterprise cannot actively perceive the occurrence of a fault and cannot carry out the initiative repair.

(2) At present, power supply enterprises have systems such as distribution automation, which can monitor the running state of the power grid in real time and locate faults. However, the traditional passive repair mode fails to take advantage of this state awareness, resulting in a waste of resources.

To sum up, the traditional model of passive repair, which is reported by electricity customers, can no longer meet the demand of current customers for high service quality nor does it make use of the current smart grid monitoring capability of power supply enterprises. Therefore, it is necessary to develop an approach based on the big data perception and intelligent processing of the power distribution networks, ${ }^{(5)}$ and to propose the initiative repair model based on the BP neural network, which derives the development of the power distribution network. Its flow chart of active rush repair is shown in Fig. 1. By adding two new links of "repair report and early warning" and "work order analysis", the initiative of the original process has been improved and a closed loop of the rush repair process has been formed.

\section{Technical Structure of Data Acquisition and Analysis}

To train the prediction model of the BP neural network, it is necessary to extract and process the big data generated in the operation of the power grid. From the definition of the big data industry chain, the key technologies of big data include not only core technologies such as data analysis technology but also important technologies such as data management, data processing, and data visualization. The technical architecture diagram is shown in Fig. 2.

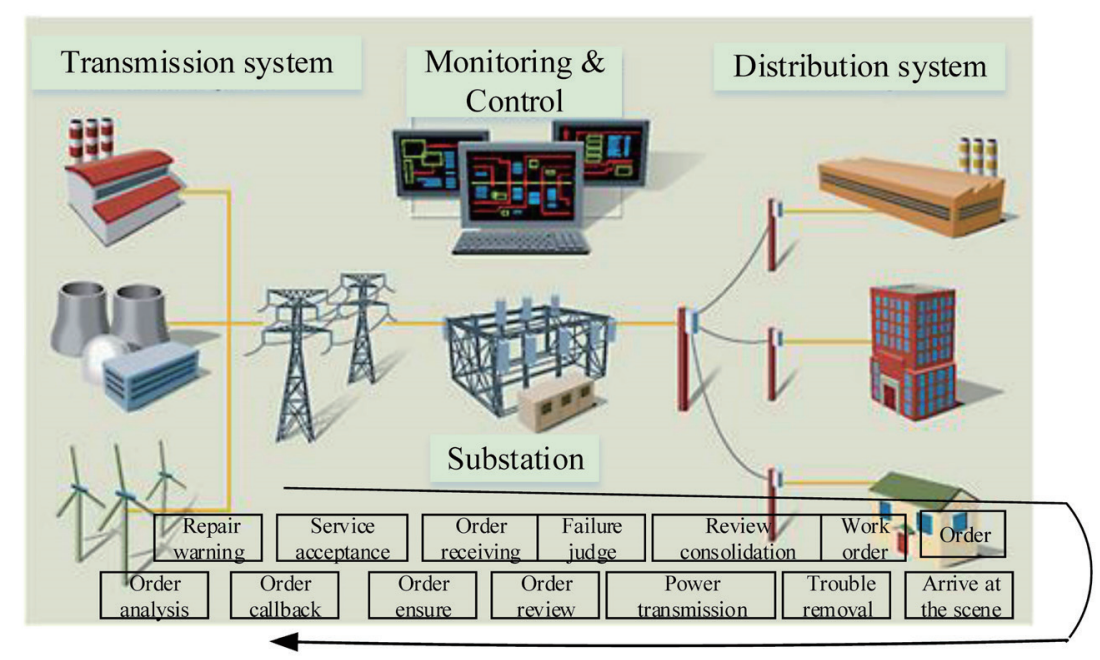

Fig. 1. (Color online) Realization of initiative repair of power distribution network. 


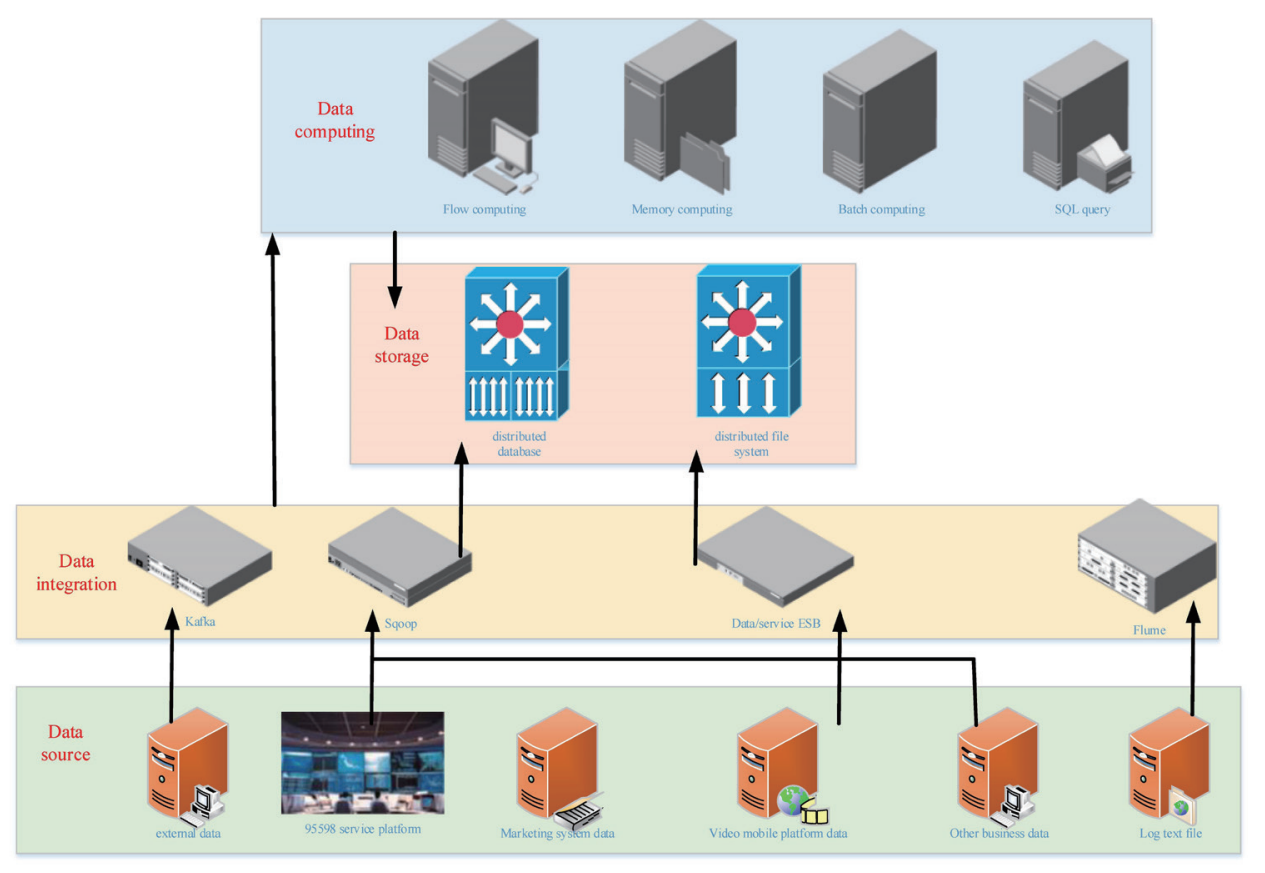

Fig. 2. (Color online) Technology architecture.

Data extraction technology: To facilitate analysis, data are mainly designed for business. Thus, it is necessary to regularly extract a large number of various types of data stored in the database of large-scale information systems and remove the incomplete data to apply it to electricity analysis. ${ }^{(6)}$ The data of this system are mainly derived from engineering production management, customer service telephone repair, real-time monitoring, grid line acquisition, monitoring assistive acquisition, history failure acquisition, application data, and so forth. After extraction, cleaning, and transformation, four processes are used for BP neural network model establishment and troubleshooting.

Data analysis technology: The comprehensive early warning and judgment of faults find and express regularities in massive data. Major methods for fault prediction include classification, clustering, correlation, and so forth. ${ }^{(7)}$ In this paper, the BP neural network is mainly used for fault analysis and prediction. This method can greatly enhance the operation and maintenance of the power distribution network.

Data service technology: To improve the speed of data access, cache memory is established in the data service database, various data analysis output interfaces are set up to provide various data access services, and all types of fault that may occur in the current power grid equipment can be displayed in the client in real time and intuitively.

Data presentation techniques: The full-type and multidimensional display of the data is a new grid fault risk in the geographic information system.

System security: Security is the lifeline of the information system. ${ }^{(8)}$ To effectively prevent attacks of illegal intruders and hackers, and protect the security of the system, a complete information security system is established by employing internal and external network separation systems in accordance with business characteristics. 
Technical safety and management safety are the two main components of an application security system. Technical security design includes application, data, system, and network security, and application security is the center of business security protection. ${ }^{(9)}$ The safety management includes security personnel, safety management system, safety technology improvement, safety assessment, supervision, and so forth. ${ }^{(10)}$

\section{Materials and Methods}

For different subclasses, the category and characteristics of data are not the same, and different training sets are required to train the fault category. Different test sets are used to predict the fault, and the failure incidence is calculated. The model is applied to the establishment of a model of the BP neural network, thereby achieving the early study of the fault. By summarizing the causes of historical failures, the cause of the fault is divided into four major categories, namely, external human factors, operation and maintenance accidents, natural disasters, and power grid equipment failures. Each type of fault can be divided into several subclasses.

The power grid equipment faults can be divided into the ring network cabinet fault, cable fault, distribution room fault, switch cabinet fault, tower fault, arrester fault, insulator fault, and so forth. The external human factors can be divided into construction damage, vehicle accidents, human theft, and so forth. Natural disaster factors can be divided into lightning, wind, flood, debris flow, and snow backlog. Operation and maintenance accidents can be divided into small animal damage, tree barrier, negligent inspection, overload voltage, and so forth.

\subsection{Study and judge model}

As shown in Fig. 3, the faults detection model mechanism includes the detection of faults reported by the customer service center and those automatically perceived by the system. On the basis of the 95598 customer repair information, electricity information acquisition system, and power distribution automation, the information is collected, extracted, cleaned, transformed, and loaded. In addition, the specific fault information is integrated to analyze the relationship among multiple faults, the probability of equipment failure, and the planned power failure information, and to comprehensively determine the fault type.

\subsection{Study and judge model procedure}

(1) Collect fault-related messages from the customer service center, electricity information acquisition system, and distribution automation platform, and remove bad data using data quality assessment.

(2) The fault information is input into the BP neural network for model training and then used in the advanced prediction of power distribution network faults in the future. Filtering scheduled power outages caused by the user generates a repair, resulting in an urgent repair work order.

(3) Retrieve and analyze the fault stations corresponding to the repair work order according to 


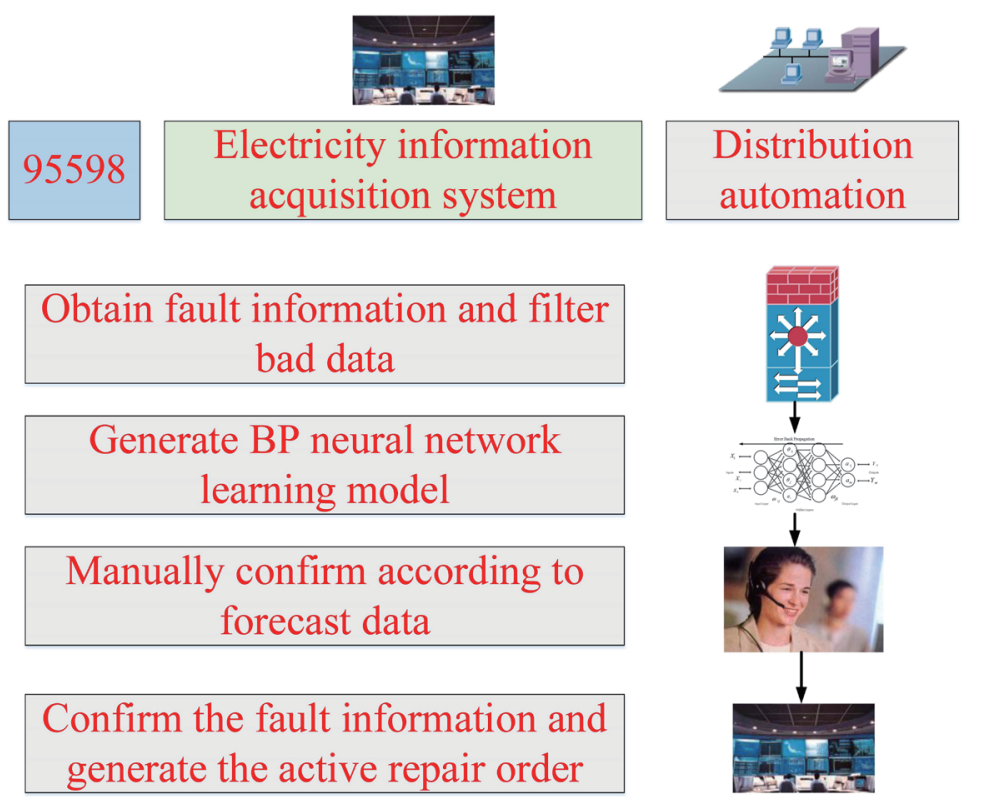

Fig. 3. (Color online) Fault study and judge model.

the fault messages of telephone repair by users, and merge the redundant urgent repair work orders in the same station area.

(4) The 95598 customer service telephone started the work order, and fault prediction sensing the active work order may be repeated, so the system conducts comprehensive research and judgment on the fault information and filters the repeated fault information to avoid repeated orders and increased workload. The system detection work order is related to the user repair work order, users are notified in various ways, the priority is given to complete the user repair work order, and the system detection work order schedule is completed.

\subsection{User initiative repair warning based on BP neural network}

In view of equipment faults, we established the fault prediction model of the power grid equipment itself from real-time monitoring data of equipment, operation data, equipment manufacturer, equipment operating life, and historical fault times of equipment, and according to historical fault information. For example, for ring network cabinet equipment, real-time monitoring data include temperature, humidity, local discharge, cable temperature, bus pile head temperature, arrester discharge times, smoke concentration, and other dimensions; operation data include voltage, current, and other dimensions; equipment manufacturer includes manufacturer name, equipment batch, and other dimensions. The collected information is shown in Table 1.

The BP neural network is one of the most widely used and successful neural networks at present. It has a simple structure, strong plasticity, and good ability to approximate nonlinear mapping. ${ }^{(11,12)}$ Therefore, it is very suitable for the initiative repair research and judgment of the power distribution network. 
Table 1

Collected Information.

\begin{tabular}{cccc}
\hline Number & Variable & Number & Variable \\
\cline { 3 - 4 } 1 & Air temperature & 2 & Air humidity \\
3 & Partial discharge & 4 & Wire temperature \\
5 & Bus pile head temperature & 6 & Discharge times of lightning rod \\
7 & Smoke concentration & 8 & Concentration of flammable gas \\
9 & Voltage & 10 & Electric current \\
11 & Manufacturer no. & 12 & Equipment production batch \\
13 & Service life of equipment & 14 & Frequency of equipment failure \\
\hline
\end{tabular}

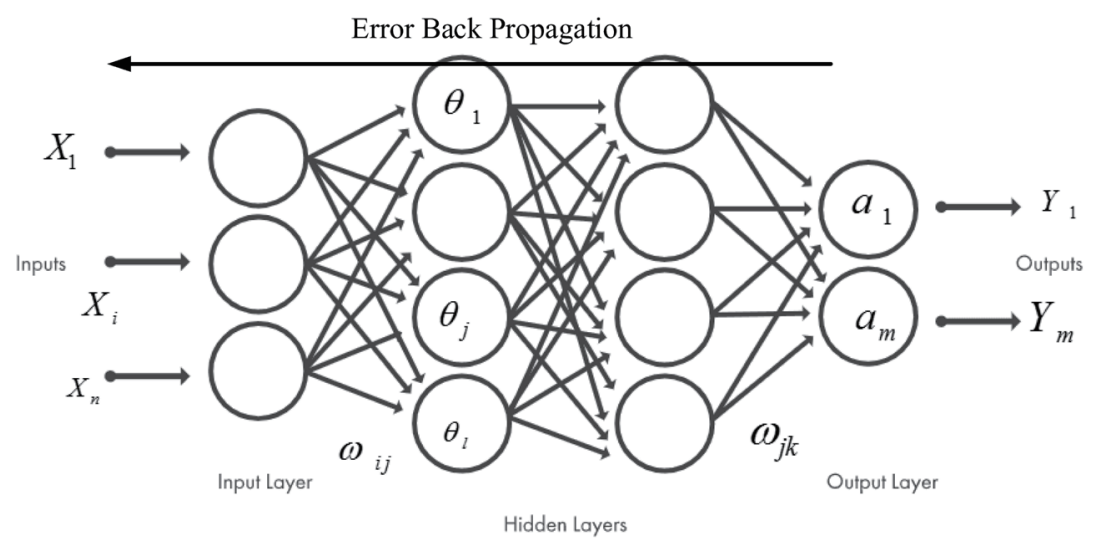

Fig. 4. (Color online) BP neural network architecture.

As shown in Fig. 4, $n$ represents the number of nodes in the input layer, $l$ defines the number of neurons in the hidden layer, $m$ is the number of neurons in the output layer, $w_{j i}$ represents the connection right between the $j$ th neuron in the hidden layer and the $i$ th input node, and $w_{k j}$ is the connection right between the $k$ th neuron in the output layer and the $j$ th neuron in the hidden layer. Then, the output of the hidden layer is

$$
H_{j}=f\left(\sum_{i=1}^{n} w_{j i} X_{i}-a_{j}\right), j=1,2, \ldots, l,
$$

where $a_{j}$ represents the threshold of the $j$ th neuron in the hidden layer and $f(\cdot)$ is a nonlinear activation function: $f(x)=1 /\left(1+e^{-x}\right)$. Therefore, the actual output of the output neuron is

$$
H_{j}^{*}=f\left(\sum_{k=1}^{n} w_{j k} * b_{k}\right), j=1,2, \ldots, l,
$$

where $b_{k}$ represents the threshold of the $k$ th neuron in the output layer.

The prediction error can be obtained from the target and actual outputs of the network. 


$$
e_{k}=\frac{1}{2} \sum_{k=1}^{m}\left(O_{k}-Y_{k}\right)^{2}
$$

In the learning process, the weight and threshold should be adjusted continuously to reduce $e_{k}$. The weight update expression is defined as follows.

$$
\begin{aligned}
& w_{k j}=w_{k j}+\eta O_{k}\left(1-O_{k}\right)\left(Y_{k}-O_{k}\right) H_{j}, j=1,2, \cdots, l ; k=1,2, \cdots, m \\
& w_{j i}=w_{j i}+\eta H_{j}\left(1-H_{j}\right) X_{i} \sum_{k=1}^{m} w_{k j} O_{k}\left(1-O_{k}\right)\left(Y_{k}-O_{k}\right), i=1,2, \cdots, n ; j=1,2, \cdots, l
\end{aligned}
$$

The threshold update expression is defined as follows.

$$
\begin{aligned}
& b_{k}=b_{k}+\eta O_{k}\left(O_{k}-1\right)\left(Y_{k}-O_{k}\right), k=1,2, \cdots, m \\
& a_{j}=a_{j}+\eta H_{j}\left(H_{j}-1\right) \sum_{k=1}^{m} w_{k j} O_{k}\left(1-O_{k}\right)\left(Y_{k}-O_{k}\right), j=1,2, \cdots, l
\end{aligned}
$$

Here, $\eta$ defines learning efficiency, and its value varies from 0 to 1 .

\section{Results and Discussion}

The numbers of layers and nodes in the hidden layer directly affect the performance of the BP neural network. ${ }^{(13)}$ To improve the prediction accuracy, on the basis of the existing results, the golden section ${ }^{(14)}$ and gradient descent methods are further used to optimize the number of hidden layer nodes in the neural network. ${ }^{(15)}$ The calculation steps are as follows:

According to the number of input nodes $\left(n_{i}\right)$ and the number of output nodes $\left(n_{0}\right)$, the following formula is used to determine the number of nodes in the hidden layer:

$$
n_{h}=\frac{n_{i} n_{o}+\frac{1}{2} n_{o}\left(n_{i}^{2}+n_{i}\right)-1}{n_{i}+n_{o}} .
$$

Assuming that $2^{k} \times n_{h}(k=1,2, \ldots)$ is regarded as the number of hidden layer nodes, selecting a small number of learning samples, and using a specific training method, we can start training learning samples according to the activation function in Eq. (1) and calculate the error. In Eq. (7), err ${ }_{a}$ represents the error of the network with the number of nodes $a$ in the hidden layer under certain conditions, and $D_{a-b}$ represents the error difference between the network with the number of nodes $a$ in the hidden layer and the network with the number of nodes $b(a<b)$ in the hidden layer under certain conditions, i.e., 


$$
D_{a-b}=\frac{e r r_{a}-e r r_{b}}{e r r_{a}}
$$

We can calculate the degree of error reduction when the numbers of hidden layer nodes are $2^{k} \times n$ and $2^{k+1} \times n$, and find the range with the largest error, which is denoted as $\left(m_{1}, m_{2}\right)$. Considering $n=0.618 \times\left(m_{1}-m_{2}\right)+m_{1}$, we determine the corresponding degrees of error reduction $D_{m_{1}-n}$ and $D_{n-m_{2}}$ in the hidden layer nodes with $n$.

If $D_{m_{1}-n}$ and $D_{a}$ are related to the required error accuracy, generally optional $0.2<D_{a}<0.4$, then $n=m_{1}$. If $m_{2}-m_{1}<\lambda \times m_{1}$, the calculation is completed, otherwise $D_{a}=0.618 \times D_{a}$; if $D_{n-m_{1}} \leq D_{a}$, then $n=m_{2}, D_{a}=0.618 \times D_{a}$; if $m_{2}-m_{1}<\lambda \times m_{1}$, the computation is finished.

After several steps of the above method, the number of nodes in the hidden layer of the optimized BP neural network can be calculated, which can be applied to the initiative repair and early warning.

According to 4659 original data samples collected, the fault prediction model of the existing BP neural network is trained. Then, a verification set was established for testing, and the results of comparing the verification data with the real data are shown in Fig. 5.

The optimized BP neural network is used to predict the faults of the power distribution network. As shown in Fig. 5, the average prediction accuracy can reach 94.5\%, basically meeting the requirements of fault prediction.

The decision tree, BP neural network, and support vector machine (SVM) algorithm were used to eliminate and predict the fault risk level, and the accuracy and recall rate of the four cases of prediction are shown in Table 2. Level 1 represents the failure of the power distribution network, i.e., slight; level 2 means important; level 3 means severe. As shown in Table 2, the accuracy rate, recall rate, and comprehensive classification performance of the BP neural network algorithm in this paper are 90.5, 80.9, and 84.2\%, respectively. Although the predicted recall rate of the decision tree algorithm for level 1 is $100 \%$, the recall rate for level 2 is $58.8 \%$,

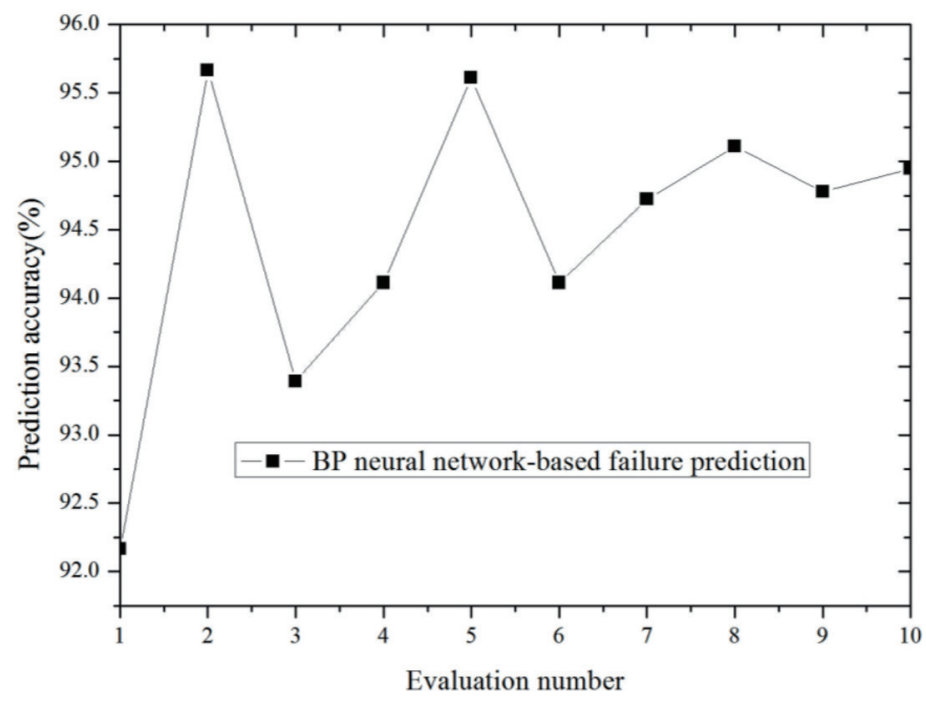

Fig. 5. Fault prediction accuracy of power distribution network. 
Table 2

Comparison of results of different classification algorithms.

\begin{tabular}{lcccccr}
\hline Method & Reference & Index & Level 1 & Level 2 & Level 3 & Accuracy \\
\hline \multirow{2}{*}{ Decision tree } & \multirow{2}{*}{$(16)$} & Precision & 75 & 90.9 & 73.7 & 78.9 \\
& & Recall & 100 & 58.8 & 85.7 & - \\
\hline \multirow{2}{*}{ BP neural } & \multirow{2}{*}{ Proposed } & Precision & 82.6 & 83.3 & 85 & 84.2 \\
\cline { 3 - 6 } & \multirow{2}{*}{ SVM } & Recall & 90.5 & 78.4 & 80.9 & - \\
& \multirow{2}{*}{$(17)$} & Precision & 83 & 86.4 & 85.7 & 85.1 \\
& & Recall & 92.9 & 78.4 & 85.7 & - \\
\hline
\end{tabular}

indicating that the adaptability of level 2 is very poor. Experimental results show that the classification performance of the fault level prediction method is better than that of the decision tree method.

\section{Conclusions}

From the background of the comprehensive construction for the ubiquitous electric Internet of Things, both failure research study and judgment location, and initiative repair of the power distribution network based on the BP neural network have been actively carried out. Through the full sensing data collection of the power distribution network and reliance on the distribution topology relationship, the fault logic algorithm is studied, which realizes not only the precise fault location of a single household, but also the intelligent fault identification of customers, precise policy and initiative repair report, and timely notification of power failure information. The quality and efficiency of fault processing and customer satisfaction are significantly improved. On the basis of the characteristics of state awareness, we designed a power distribution network based on the BP neural network to elucidate the initiative repair mechanism. The method can give full attention to the technical advantage of the BP neural network method, and compared with the traditional fault analysis method, this method realizes the initiative repair of the power distribution network, greatly shortens the time of troubleshooting, and improves the efficiency and accuracy of the fault repair research. Moreover, the accuracy of fault diagnosis is improved. This method effectively promotes the transformation of the initiative repair mode of the power distribution network from passive to active and significantly improves the service quality perceived by customers.

\section{Acknowledgments}

This work was supported by the National Natural Science Foundation of China (Grant 51577028).

\section{References}

1 H. Zayandehroodi, A. Mohamed, H. Shareef, and M. Mohammadjafari: J. Appl. Sci. 10 (2010) 3032. https://doi. org/10.3923/jas.2010.3032.3041

2 Y. Wei, P. Sun, Z. Song, P. Wang, Z. Zeng, and X. Wang: IEEE Access 9 (2021) 31246. https://doi.org/10.1109/ ACCESS.2021.3059935 
3 H. Sun, Y. Zhang, and B. Hu: Cluster Comput. 22 (2019) 5293. https://doi.org/10.1007/s10586-017-1225-5

4 M. Schmitz, D. P. Bernardon, V. J. Garcia, W. I. Schmitz, M. Wolter, and L. L. Pfitscher: IEEE Trans. Smart Grid 12 (2021) 324. https://doi.org/10.1109/TSG.2020.3018640

5 A. Arefi, G. Ledwich, G. Nourbakhsh, and B. Behi: IEEE Trans. Smart Grid 11 (2020) 3896. https://doi. org/10.1109/TSG.2020.2977211

6 T. Mathes, P. Klaßen, and D. Pieper: BMC Med. Res. Methodol. 17 (2017) 152. http://doi.org/10.1186/s12874017-0431-4

7 Y. Qian, X. Liang, Q. Wang, J. Liang, B. Liu, A. Skowron, Y. Yao, J. Ma, and C. Dang: Int. J. Approximate Reasoning 97 (2018) 38. https://doi.org/10.1016/j.ijar.2018.01.008

8 I. Doric, D. Arp, S. Werr, and H. P. Schoener: ATZ Worldwide eMag. 121 (2019) 58. https://doi.org/10.10.1007/ s38311-018-0186-5

9 S. Y. R. Hui: IEEE Electromagn. Compat. Mag. 7 (2018) 78. https://doi.org/10.1109/MEMC.0.8339551

10 S. N. Trostyansky, A. V. Kalach, V. V. Lavlinsky, and O. V. Lankin: J. Phys.: Conf. Ser. 973 (2018) 012049. https://doi.org/10.1088/1742-6596/973/1/012049

11 F. He and L. Zhang: J. Process Control. 66 (2018) 51. https://doi.org/10.1016/j.jprocont.2018.03.005

12 S. Geng and X. Wang: Comput. Electr. Eng. 86 (2020) 106732.https://doi.org/10.1016/j.compeleceng.2020.106732

13 H. Zheng, X. Tuo, S. Peng, R. Shi, H. Li, J. Lu, and J. Li: Nucl. Sci. Tech. 29 (2018) 61. https://doi.org/10.1007/ s41365-018-0410-4

14 A. N. Samudrala, M. H. Amini, S. Kar, and R. S. Blum: IEEE Trans. Smart Grid 11 (2020) 5124. https://doi. org/10.1109/TSG.2020.2999921

15 K. Shujaat, N. Imran, M. M. Ammar, T. Roberto, and B. Mohammed: Circuits Syst. Signal Process. 37 (2018) 5311. https://doi.org/10.1007/s00034-018-0835-3

16 S. Ghimire and S. M. Alizadeh: Energies 14 (2021) 2293. https://doi.org/10.3390/EN14082293

17 Y. Hamid, L. Journaux, J. A. Lee, and M. Sugumaran: Int. J. Artif. Intell. Soft Comput. 6 (2018) 265. https://doi. org/10.1504/IJAISC.2018.097280

\section{About the Authors}

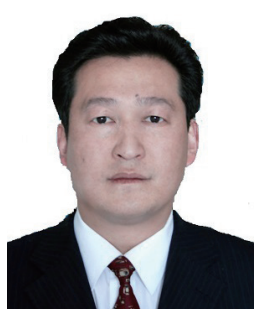

Zhihua Guo received his B.S. degree in Electronic Instruments and Measuring Technique from Changchun University of Science and Technology, China in 1997. He joined State Grid Qinghai Electric Power Co., Ltd., where he is currently a senior engineer. His research interests are in power marketing and power metering. (gzh7028@163.com.cn)

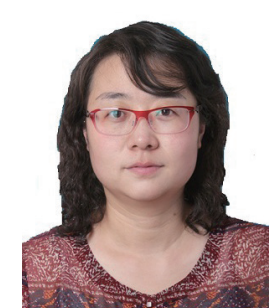

Na Li received her B.S. degree from Changchun University of Technology, China, and Master's degree from the University of Electronic Science and Technology of China (UESTC), China, in 2003 and 2012, respectively. She joined State Grid Qinghai Electric Power Co., Ltd., where she is currently a senior engineer focusing on power engineering technology. Her research interests are in online loss and electric energy metering and collection. (lena110@126.com.cn)

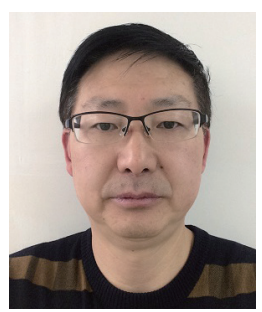

Dongping Qiao received his B.S. degree in Computer Application from Northwest Institute of Telecommunication Engineering, China, in 1987. He joined State Grid Qinghai Electric Power Co., Ltd., where he is currently a senior engineer focusing on industrial engineering. His research interests are in power operation management and power marketing. (syyxzzbjb@126.com) 


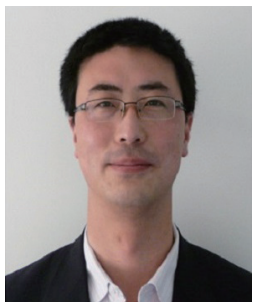

Hu Qiao received his B.S. degree in Computer Science and Technology from Lanzhou University, China in 2001. He joined State Grid Qinghai Electric Power Co., Ltd., where he is currently a senior engineer focusing on industrial engineering. His research interests are in electricity price and electricity and energy metering. (qiaohu4251@qh.sgcc.com.cn)

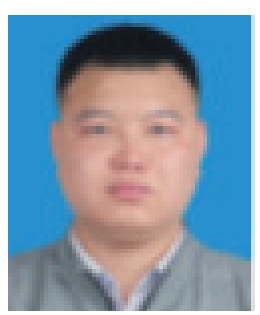

Chao He received his B.S. and M.S. degrees from Chongqing Three Gorges University in China in 2014 and 2017, respectively. Now, he is currently pursuing his Ph.D. degree from School of Communication and Information Engineering, Chongqing University of Posts and Telecommunications. His research interests are in MEC, VNE, and FiWi broadband access network. (d170101004@stu.cqupt.edu.cn) 